\title{
Effect of mesh type, surgeon and selected patients' characteristics on the treatment of inguinal hernia with the Lichtenstein technique. Randomized trial
}

\author{
Konrad Pielaciński ${ }^{1}$, Andrzej B. Szczepanik ${ }^{1}$, Tadeusz Wróblewski² \\ 1Department of General and Hematological Surgery, Institute of Hematology and Transfusion Medicine, Warsaw, Poland \\ 2Department of General, Transplant and Liver Surgery, Warsaw Medical University, Poland
}

Videosurgery Miniinv 2013; 8 (2): 99-106 DOI: 10.5114/wiitm.2011.32824

\begin{abstract}
Introduction: Though not entirely free of complications, the Lichtenstein technique is still considered the "gold standard" for inguinal hernia repair due to the low recurrence rate.

Aim: In our study we determined the effect of mesh type, surgeon and selected patients' characteristics on treatment results. The latter were determined by the frequency of early complications, recovery time and return to normal activities, chronic pain and hernia recurrence.

Material and methods: Tension-free hernia repair with the Lichtenstein technique was performed in 149 male patients aged 20-89 years randomized to two trial groups. One group comprised 76 patients with heavyweight non-absorbable polypropylene mesh (HW group) and the other included 73 patients with lightweight partially absorbable mesh (LW group). The control schedule follow-up took place on the $7^{\text {th }}$ day as well as in the $3^{\text {rd }}$ and $6^{\text {th }}$ month after the operation. Statistical analysis was performed with multi-factor regression models.

Results: In the LW group patients returned to normal activity faster ( $p=0.031)$, experienced less intensive chronic pain $(p=0.01)$ and expressed higher treatment satisfaction $(p=0.024)$ than the patients from the HW group. The type of mesh had an insignificant influence on the risk of early complications and hernia recurrence. Statistically significant differences were observed however with regard to surgeon, type and hernia duration, patient's general condition and body mass.

Conclusions: Both types of mesh are equally effective for prevention of hernia recurrence. Lightweight partially absorbable meshes are more beneficial to patients than the heavyweight non-absorbable type. The surgeon and patients' characteristics have a significant impact on the treatment outcome.
\end{abstract}

Key words: inguinal hernia, Lichtenstein technique, non- and partially absorbable mesh.

\section{Introduction}

The common tension-free surgical techniques currently used for inguinal hernia repair fall into two categories with regard to placement of the mesh in relation to the transverse fascia: anterior (e.g. Licht- enstein technique) and posterior with the mesh placed behind the transverse fascia utilizing Nyhus, Stoppa open technique or video-assisted totally extraperitoneal (TEP) or trans-abdominal pre-peritoneal (TAPP) technique [1-5]. For primary inguinal 
hernia repair however the most frequent treatment of choice is the open Lichtenstein method, which is relatively uncomplicated and highly effective for prevention of hernia recurrence [1, 2, 6-8]. Although implants of synthetic material have brought long awaited improvement in hernia management regardless of the method used and have reduced hernia recurrence to $1-2 \%$, the tension-free techniques are not free of adverse effects [4, 6, 7, 9-11]. Chronic pain in the operated groin remains an unsolved clinical problem reported in about $11 \%$ of patients and in $8 \%$ of them pain intensity affects the quality of everyday life [12-14]. The most likely causes of chronic pain, apart from surgical trauma related to hernioplasty and technical accuracy [8-10, 15, 16], are the psychological and somatic characteristics of patients $[12,17]$ as well as the type of mesh and placement site $[14,18-21]$. It is common opinion that traditional heavyweight polypropylene meshes may cause more severe pain complications than the lightweight partially absorbable meshes $[18,20,21]$. This however has not been univocally confirmed in studies to date, particularly those with long-term follow-up [22, 23].

\section{Aim}

The primary aim of the study was to determine the effect of type of mesh (heavyweight non-absorbable mesh vs. lightweight partially absorbable mesh) used for inguinal hernia repair with Lichtenstein technique on the risk of hernia recurrence, chronic postoperative pain, postoperative complications such as feeling of a foreign body, recovery time, return to normal activities and sport, intensity of postoperative pain and analgesic consumption as well as patients' satisfaction with treatment.

The secondary aim was to evaluate the effect of surgeon and selected patients' characteristics such as type of hernia, hernia duration, body mass index (BMI) as well as general condition (ASA) on the above-mentioned parameters.

\section{Material and methods}

The study comprised men over 18 years of age admitted for elective surgery of primary or recurrent inguinal hernia (following tension techniques) and no other changes in the groin or scrotum. All patients gave their written consent for participation in the study. The study protocol and aims were approved by the Bioethi- cal Committee of the Institute of Hematology and Transfusion Medicine in Warsaw (9/2008). One hundred and seventy-six patients were randomized to two study groups. All inclusion criteria were fulfilled by 149 men aged 23 to 89 years. The HW group comprised 76 men operated on by the Lichtenstein technique with a typical heavyweight non-absorbable mesh (polypropylene; $1 \mathrm{~mm}$ pore size, $100 \mathrm{~g} / \mathrm{m}^{2}$ weight) and the LW group included 73 men with lightweight, partially absorbable composite mesh (4 $\mathrm{mm}$ pores and primary weight of $80 \mathrm{~g} / \mathrm{m}^{2}$ consisting of nonabsorbable polypropylene fiber $\left(35 \mathrm{~g} / \mathrm{m}^{2}\right)$ and absorbable polyglactin $910\left(45 \mathrm{~g} / \mathrm{m}^{2}\right)$. Characteristics of both groups are presented in Table I. The Liechtenstein technique [1] was modified so that a 2.0 polypropylene continuous fixing suture was applied from both the upper and the lower mesh edge and then a vacuum drain (Redon) was inserted through a separate percutaneous incision. The procedure was obligatorily performed under subarachnoid anesthesia ( $0.5 \%$ bupivacaine hydrochloride) with antibacterial (cefazolin 1.0 g, i.v.) and antithrombotic prophylaxis (nadroparin $0.3 \mathrm{ml}$, s.c.). The type of hernia was determined intraoperatively and the Nyhus classification was applied. In the case of bilateral hernia two separate procedures were performed. Hernia which was more disturbing was operated on first and hernia on the other side after at least a month following the first surgery. Such patients were treated as patients with one-sided hernias manifesting bilateralhernia characteristics. Study data were collected according to the study protocol during hospitalization and scheduled follow-up appointments after the $7^{\text {th }}$ day as well as the $3^{\text {rd }}$ and $6^{\text {th }}$ month of surgery. Ketoprofen was used as an analgesic, and pain intensity was determined on a visual analogue scale (VAS). After $24 \mathrm{~h}$ patients were allowed to walk. They were usually released from hospital not earlier than on the $1^{\text {st }}$ or $2^{\text {nd }}$ postoperative day. They were recommended to restrain from driving until the $7^{\text {th }}$ day and bike riding until the $14^{\text {th }}$ day; lifting heavy objects $>10 \mathrm{~kg}$ was allowed after 3 months. Satisfaction with treatment was based on patients' subjective assessment of comfort following hernia removal (surgical result) and scar appearance (plastic result). The scale included the following options: very good, good, acceptable and bad.

\section{Statistical analysis}

Patients' characteristics (Table I) were compared using the $\chi^{2}$ test or Fisher's exact test as well as Stu- 
Table I. Characteristics of randomized patients who fulfill all inclusion criteria for surgery with nonabsorbable mesh (HW group) or partially absorbable mesh (LW group)

\begin{tabular}{|c|c|c|c|c|}
\hline Parameter & $\begin{array}{l}\text { HW group } \\
(n=76)\end{array}$ & $\begin{array}{l}\text { LW group } \\
(n=73)\end{array}$ & $\begin{array}{c}\text { Total no. of patients } \\
(n=149)\end{array}$ & Value of $p$ \\
\hline Age, average (range) & $59(20-89)$ & $58(24-87)$ & $59(20-89)$ & 0.5129 \\
\hline Type of hernia (acc. to Nyhus): & & & & 0.477 \\
\hline I & $10(13 \%)$ & $12(16 \%)$ & $22(15 \%)$ & - \\
\hline$\|$ & $32(42 \%)$ & $27(37 \%)$ & $59(40 \%)$ & - \\
\hline$\overline{I I I}$ & $33(44 \%)$ & $30(41 \%)$ & $63(42 \%)$ & - \\
\hline IV & $1(1 \%)$ & $4(5 \%)$ & $5(3 \%)$ & - \\
\hline Hernia: & & & & 0.227 \\
\hline Left side hernia & $30(40 \%)$ & $36(50 \%)$ & $66(44 \%)$ & - \\
\hline Right side hernia & $46(61 \%)$ & $37(51 \%)$ & $83(56 \%)$ & - \\
\hline Bilateral & $16(21 \%)$ & $16(22 \%)$ & $32(21 \%)$ & 0.898 \\
\hline ASA score: & & & & 0.771 \\
\hline I & $30(40 \%)$ & $32(45 \%)$ & $62(42 \%)$ & - \\
\hline$\overline{11}$ & $34(45 \%)$ & $28(40 \%)$ & $62(42 \%)$ & - \\
\hline III & $12(16 \%)$ & $11(15 \%)$ & $23(16 \%)$ & - \\
\hline Body mass index (BMI): & & & & 0.800 \\
\hline Proper body mass & $40(53 \%)$ & $34(48 \%)$ & $74(50 \%)$ & - \\
\hline Excess weight & $27(36 \%)$ & $29(41 \%)$ & $56(38 \%)$ & - \\
\hline Obesity & $9(12 \%)$ & $8(11 \%)$ & $17(12 \%)$ & - \\
\hline $\begin{array}{l}\text { Hernia duration [weeks] } \\
\text { median (range) }\end{array}$ & $67(1-2400)$ & $48(1-1920)$ & $48(1-2400)$ & 0.200 \\
\hline Previous surgery & $44(58 \%)$ & $44(62 \%)$ & $88(60 \%)$ & 0.614 \\
\hline Concurrent diseases & $44(58 \%)$ & $40(56 \%)$ & $84(57 \%)$ & 0.849 \\
\hline Surgeon: & & & & 0.007 \\
\hline 1 & $40(54 \%)$ & $56(79 \%)$ & $96(66 \%)$ & \\
\hline 2 & $16(22 \%)$ & $7(10 \%)$ & $23(16 \%)$ & \\
\hline Others & $18(24 \%)$ & $8(11 \%)$ & $26(18 \%)$ & \\
\hline
\end{tabular}

dent's $t$ test or non-parametric Mann-Whitney test. The standard set of variables used in the regression models included: mesh type, hernia type, body mass determined by BMI, functional status according to ASA scale, hernia duration (categorized as median of 48 weeks), interactions between the mesh and hernia type, previous surgery (yes/no), surgeon (1, 2, others), and bilateral hernia indicator. Logistic regression models were used to analyze: surgery duration, intraoperative damage, immediate postoperative complications in the groin and/or scrotum, return to normal activities of daily living and sport, analgesic consumption up to 8 days after surgery. The analysis of severe postoperative pain intensity was based on a standard set of variables extended by such variables as time trend, quadratic trend and the relationship between them. The significance of random effects in the model was tested with mixed models of $\chi^{2}$ with various values of diversity. For analysis of analgesic consumption and the values on the pain scale we used the mixed model explaining the level of the parameter by means of fixed and random effects which took into account patients' individual diversity. The significance of fixed effects and the type of matrix correlation for individual time points was tested with the restricted maximum likelihood (REML) method. The standard set of variables was extended by age and used for the analysis of pain 
intensity in the $3^{\text {rd }}$ and $6^{\text {th }}$ postoperative month using Poisson regression models, the risk of ailments in the $3^{\text {rd }}$ and $6^{\text {th }}$ postoperative month using logistic regression models, and degree of impairment in quality of daily activity as a result of ailments was examined with the Poisson model. We examined patients' satisfaction with treatment using the ordinal regression model with a standard set of variables extended by pain incidence and ailments in the $3^{\text {rd }}$ and $6^{\text {th }}$ postoperative month. In all the tests the statistical significance was assumed at $p \leq 0.05$.

\section{Results}

No statistically significant differences in patients' characteristics were observed between HW and LW groups (Table I).

Early complications occurred in 44 of the 149 surgically treated patients (29.5\%). These included: wound hematoma in 22 patients (14.7\%), wound infection in $2(1.2 \%)$, retention of urine requiring catheterization in $10(6.7 \%)$, and 3 for each of groin seroma, ischemic orchitis and headaches following subarachnoid anesthesia (2\%). No statistically significant effect of mesh type on the risk of early complications was observed. However, patients in ASA class III presented a 5 -fold higher risk of early complications $(\mathrm{OR}=5.235,95 \% \mathrm{Cl}$ $=1.368$ to 20.032; $p=0.016$ ) and patients operated on by surgeons other than 1 and 2 presented a 3.5 -fold risk $(\mathrm{OR}=3.658,95 \% \mathrm{Cl}=1.263$ to $10.591 ; p=0.017)$. Four cases $(2.87 \%)$ of hernia recurrence were observed: 2 for each type of mesh. In the group with nonabsorbable mesh it was $2.81 \%$ of all operated patients; in the group with partially absorbable mesh it was $2.94 \%$. No significant effect of mesh type on the risk of hernia recurrence was observed $(\mathrm{OR}=0.97,95 \% \mathrm{Cl}=$ 0.132 to $7.093 ; p=0.977$ ).

In all, 39 patients were excluded from the $149 \mathrm{pa}-$ tients randomized into the two study groups: 22 (29\%) from the group with non-absorbable mesh and 17 (23\%) from the group with partially absorbable mesh ( $p=$ 0.432 ). Exclusion criteria were as follows: intraoperative damage of anatomical structures in $9(27 \%)$ patients, including 4 with HW mesh and 5 with LW mesh, postoperative complications and/or worsening of general condition in 16 (41\%) patients (HW 7, LW 9), hernia recurrence in 4 (2.87\%) (HW 2, LW 2) and failure to appear for scheduled follow-up visits in $10(25 \%)$ patients (HW 7, LW 3).
One hundred and ten patients with an uneventful course of treatment were qualified for further evaluation: 54 with non-absorbable mesh (HW) and 56 with partially absorbable mesh (LW). No significant effect of the mesh type on surgery duration was observed. Surgery performed by surgeon 2 was shorter by 9 min on average as compared to surgery performed by other surgeons ( $B=-9.259,95 \% \mathrm{Cl}=-14.818$ to -3.699 ; $p<0.001)$. The condition of ASA III patients prolonged surgery duration by $9 \mathrm{~min}$ on average $(B=9.439$, $95 \% \mathrm{Cl}=3.776$ to $15.102 ; p<0.001)$ as compared to patients with other results on the ASA scale. In patients with hernia duration above 48 weeks, surgery duration was 7 min longer than in patients with shorter hernia duration $(B=7.478,95 \% \mathrm{Cl}=3.317$ to 11.639; $p<0.001)$. In 5 patients from the HW group and 4 patients from the LW group (9 in all), we observed 15 cases of damage to anatomical structures including 8 cases of damage to the iliohypogastric nerve, 6 to the ilioinguinal nerve and 1 case of damage to the spermatic duct. No significant effect of mesh type on the risk of intraoperative damage to anatomical structures was observed. However, the risk was 4.5 -fold higher $(\mathrm{OR}=4.479,95 \% \mathrm{Cl}=1.032$ to 19.435; $p=0.045$ ) for patients operated on by surgeons other than 1 or 2 . For patients with hernia type II or III the risk of intraoperative damage was about $84 \%$ lower than for patients with hernia type I (OR = $0.164,95 \% \mathrm{Cl}=0.037$ to $0.712 ; p=0.016)$, and the ASA type III evaluation resulted in a 6.5 -fold increase as compared to other evaluations on the ASA scale $(\mathrm{OR}=6.512,95 \% \mathrm{Cl}=1.302$ to $32.56 ; p=0.023)$.

During 7 postoperative days no significant effect of the type of mesh, surgeon, or patients' characteristics on the intensity of acute postoperative pain evaluated on the VAS scale was observed.

We observed a significant interaction between mesh type and type of hernia as well as result on the ASA scale on the analgesic consumption on surgery days and the $7^{\text {th }}$ postoperative day (8 days in all). The use of LW mesh increased the amount of analgesic consumption by $278 \mathrm{mg}$ on average as compared to cases in which HW mesh was used $(B=277.92,95 \% \mathrm{Cl}$ $=67.77$ to $488.07 ; p=0.010$ ). However, in cases of II, III and IV type hernias the effect of LW mesh decreased analgesic consumption by $332 \mathrm{mg}(B=-322.43,95 \% \mathrm{Cl}$ $=-536.29$ to $-108.57 ; p=0.003)$ as compared to patients with type I hernia and the same mesh type and patients with all hernia types and HW mesh. As 
a result, patients with LW mesh and hernia type I had the highest need for analgesics; they were administered $278 \mathrm{mg}$ of ketoprofen more than all patients with HW mesh and $44 \mathrm{mg}$ more than those with LW mesh and hernia type II, III or IV. Patients with partially LW mesh and hernia type II, III or IV were administered $234 \mathrm{mg}$ of ketoprofen more than all patients with HW mesh. On average, ASA III or II patients were given $130 \mathrm{mg}$ of analgesic less than the ASA I patients ( $B=$ $-130.54,95 \% \mathrm{Cl}=-237.75$ to $-23.35 ; p=0.017)$. Both in the multi-factor and one-factor analysis the interaction between type of mesh and hernia type was found to have a significant influence on return to normal activities of daily living. When LW mesh was used patients with hernia type II returned to normal living faster (by 0.006 day) ( $B=-0.006,95 \% \mathrm{Cl}=-0.013$ to -0.0002 ; $p=0.043)$ than the other patients according to multifactor analysis and by 5.02 days $(B=-5.206,95 \% \mathrm{Cl}=$ -9.914 to $-0.499 ; p=0.031$ ) according to one-factor analysis. One-factor analysis also revealed that LW mesh used for patients with hernia type III resulted in faster return to normal activities of daily living by 5.8 days as compared to the other patients $(B=-5.817$, $95 \% \mathrm{Cl}=-10.502$ to $-1.133 ; p=0.015$ ). Moreover, patients operated on by surgeon 2 returned to normal activities of daily living faster (by about 2.5 days) ( $B=-2.398,95 \% \mathrm{Cl}$ $=-4.610$ to $-0.186 ; p=0.034$ ) than those operated on by other surgeons.

The recovery time for patients with bilateral hernias was shorter by 2.5 days than for patients with one-sided hernias $(B=-2.436,95 \% \mathrm{Cl}=-4.133$ to $-0.745 ; p=0.005)$. The return to normal activities was faster by almost 2 days for patients with hernia duration of over 48 weeks prior to surgery than for patients with shorter hernia duration $(B=-1.713$, $95 \% \mathrm{Cl}=-3.206$ to $-0.221 ; p=0.025)$.

The return to sport activities was almost 19 days faster for patients with LW mesh than for patients with $\mathrm{HW}$ mesh $(B=-18.852,95 \% \mathrm{Cl}=-36.919$ to $-0.785 ; p=0.001)$. No effect of the surgeon on recovery time and return to sport activities was observed. Effect of hernia type on bore parameters was as follows: patients with hernia type II or III returned to sport activities faster than those with hernia type I by 24 days $(B=-24.281,95 \% \mathrm{Cl}=-40.950$ to -7.612 ; $p=0.001)$ and with type IV by 30 days $(B=-30.118$, $95 \% \mathrm{Cl}=-46.389$ to $-13.847 ; p=0.001$ ).

Three months after surgery chronic pain in the operated groin was reported in 15/110 (13.5\%) patients including 9/54 (17\%) from the HW group and 6/56 (11\%) from the LW group; the pain was assessed as 2 to 6 on the VAS scale. After 6 months a 2 to 4 VAS scale pain was reported in 10 (9\%) patients, including 7 (12\%) from the HW group and in 3 (6\%) from the LW group. After the $3^{\text {rd }}$ and $6^{\text {th }}$ month single cases of ketoprofen administration at a dose of 50 to $100 \mathrm{mg}$ were reported. The intensity of chronic pain was by $60 \%$ lower (IRR $=0.418,95 \% \mathrm{Cl}=0.160$ to $1.092 ; p=0.075)$ after 3 months and by $80 \%(I R R=0.233,95 \% \mathrm{Cl}=0.075$ to $0.720 ; p=0.011)$ after 6 months for patients with LW mesh as compared to those with HW mesh. Moreover, after 3 months we observed a 4-fold higher intensity of chronic pain (IRR $=4.208,95 \% \mathrm{Cl}=1.714$ to 10.334 ; $p=0.002)$ in the groin operated by a surgeon other than 1 or 2 . For patients with hernia type II, III or IV the pain was 74\% less intense than for patients with hernia type I (IRR $=0.258,95 \% \mathrm{Cl}=0.136$ to $0.891 ; p=0.008)$. The intensity of chronic pain in the groin was 4-fold higher in obese and overweight patients as compared to those with normal body mass (IRR $=4.058,95 \% \mathrm{CI}=$ 0.816 to $12.416 ; p=0.003)$. After 6 months, patients with hernia type II experienced 6-fold more intense pain than the other patients ( $\mathrm{IRR}=5.767,95 \% \mathrm{Cl}=$ 1.891 to $17.594 ; p=0.002$ ).

After the $3^{\text {rd }}$ postoperative month complaints such as feeling of a foreign body, prickly or pinching sensation, tightness and pulling or other unspecified discomfort were observed in the operated groin in 54 (49\%) patients including 32 (59\%) with HW mesh and 22 (39\%) with LW mesh. After the $6^{\text {th }}$ postoperative month however, these sensations were felt by 40 (36\%) patients including 23 (43\%) with HW mesh and 17 (30\%) with LW mesh. Such sensations were observed during physical activity, both after the $3^{\text {rd }}$ and $6^{\text {th }}$ postoperative months.

A significant effect of mesh type and bilateral hernia on the risk of such sensations after the $3^{\text {rd }}$ month was observed. For patients with LW mesh the risk of these sensations during the first 3 post-operative months was $73 \%$ lower than for patients with HW mesh $(\mathrm{OR}=0.275,95 \% \mathrm{Cl}=0.112$ to $0.671 ; p=0.005)$. The risk was $84 \%$ lower for patients with bilateral inguinal hernias than for patients with one-sided hernias $(\mathrm{OR}=0.166,95 \% \mathrm{Cl}=0.058$ to 0.469 ; $p=0.001)$. After the 6 th postoperative month the effect of mesh type, patients' characteristics and surgeon were found to be statistically insignificant.

According to subjective assessment scale (very good/good/acceptable/bad) the surgical results were: 
on day 7: $95(86 \%) / 13(12 \%) / 1(1 \%) / 0$, after 3 months: $93(85 \%) / 16(15 \%) / 0 / 1(1 \%)$, and 6 months after surgery: 101 (92\%)/7 (6\%)/1 (1\%)/1 (1\%) respectively. Reports for the plastic effect were: on day 7: $82(75 \%) / 27(25 \%) / 1(1 \%) / 0$, after 3 months: 89 (81\%)/ $20(18 \%) / 1(1 \%) / 0$ and after 6 months: 98 (89\%)/ $11(10 \%) / 1(1 \%) / 0$ respectively.

On the $7^{\text {th }}$ day and after the $3^{\text {rd }}$ month none of the analyzed variables had a significant effect on the assessment of surgical effect; the surgeon was the only variable with a significant effect on the assessment of the plastic effect. On the $7^{\text {th }}$ day the chance for a better plastic effect increased by about $80 \%$ for surgery performed by surgeon 1 or $2(\mathrm{OR}=0.218$, $95 \% \mathrm{Cl}=0.065$ to $0.734 ; p=0.014)$ and after the $3^{\text {rd }}$ month this chance increased 4 times $(O R=4.275$, $95 \% \mathrm{Cl}=0.065$ to $12.717 ; p=0.009)$. After the $6^{\text {th }}$ month the surgeon and mesh type had a significant effect on the assessment of the surgical result. For patients with LW mesh we observed a 6-fold higher treatment satisfaction rate for patients with $\mathrm{HW}$ mesh $(\mathrm{OR}=6.079,95 \% \mathrm{Cl}=1.263$ to 29.253 ; $p=0.024)$. On the other hand, surgery performed by a surgeon other than 1 or 2 reduced the chance for better patient satisfaction with treatment by about $80 \%(\mathrm{OR}=0.183,95 \% \mathrm{Cl}=0.036$ to $0.923 ; p=0.039)$. None of the analyzed variables had a significant effect on the final evaluation of the plastic result after the $6^{\text {th }}$ month.

\section{Discussion}

Although tension-free surgical techniques for inguinal hernia repair have contributed to the improvement of the outcome as well as the marked reduction of hernia recurrence, these techniques are not free of complications [4, 6, 7, 9-11]. The most common and still unresolved clinical problem is the chronic pain in the operated groin. One of the likely reasons which are taken into account is the presence of the mesh and its type [12-14]. Research data show that the amount and structure of the implanted material have a significant influence on scar tissue formation. Heavy propylene non-absorbable meshes cause a more intense inflammatory reaction and scar formation than the light propylene or partially absorbable meshes [21, 24]. The implied superiority of light meshes was confirmed in clinical trials but with relatively short follow-up periods [20, 21]. Results for longer follow-up periods did not prove so convincing.
There are even reports demonstrating no significant differences between types of meshes [22, 23].

Therefore the aim of our study was to determine the effect not only of mesh type but also of the surgeon as well as selected patients' characteristics on results of inguinal hernia repair using the Lichtenstein technique. During the 6-month follow-up period we observed four hernia recurrences, two in each of the study groups (NS); therefore the risk of hernia recurrence following surgery with light, partially absorbable meshes was not higher as reported by O'Dwyer et al. [20]. The incidence rate for hernia recurrence in our study (2.87\%) is comparable to literature reports from similar studies (from $2 \%$ to $8 \%$ ) but worse than the results reported by centers for hernia repair $[1,3,4]$. The incidence rate for hernia recurrence proved insufficient for the evaluation of the effect of the surgeon and selected patients' characteristics in multifactor models.

Despite the relatively low risk of perioperative wound infection (2-4\% with or without prophylaxis respectively $[25,26])$ reported in randomized clinical trials, the benefits of antibacterial prophylaxis are unquestioned and it is therefore recommended [25]. In our study we routinely administered antibacterial prophylaxis in the form of a single dose of antibiotic, particularly for patients with additional wound infection risk factors (diabetes, drains [2, 7]). Despite these precaution measures wound infection was observed in 2 patients (1.6\%). The result is comparable to results from similar studies as well as from metaanalyses (2.3-3.4\%) [8, 9, 25-27].

The incidence rate of intraoperative damage of anatomical structures and early complications observed in 44 of our patients $(29.5 \%)$ is similar to literature data (8-22\%) $[6,9,27]$. Like some authors [8, $16,28,29]$, we found that the risk of intraoperative damage and early postoperative complications increases significantly for patients operated on by surgeons other than 1 or 2 as well as ASA III patients.

In our study no effect of mesh type on surgery duration was observed ( $\mathrm{HW}-48.8 \mathrm{~min}$ vs. $\mathrm{LW}-47.6 \mathrm{~min})$. In the literature, the results vary. Some authors report no significant influence of mesh type on surgery duration [16, 22, 23], while others have found that placement of non-absorbable mesh is shorter, which may suggest that this type is easier to fix [18]. Moreover, our study shows that surgery by surgeon 2 significantly reduced procedure duration as com- 
pared to the other surgeons. The effect of the operator on surgery duration is a well-acknowledged fact confirmed by published study results [8, 11, 30, 31].

In our study the patients returned to normal activities of daily living 5 days after surgery on average, which was consistent with results of similar studies (4-10 days on average) [11, 22]. The shorter return to normal activities of daily living and sport observed for our patients with LW mesh was also confirmed by published results from similar studies and meta-analyses that confirm the beneficial effect of light meshes [18, 21, 23, 32].

Most studies report that the use of the light meshes results in less intense pain, in both the early and the late postoperative period [20, 23]. Our observations however are consistent with those of Post et al., Koch et al., and Nikkolo et al. [21, 22, 33] and do not confirm the significant effect of mesh type (and hernia type) on pain intensity in the early postoperative period.

Analgesic consumption revealed that the total requirement for painkillers was higher for patients with LW mesh than for patients with HW mesh. This result is not consistent with results from other studies [23, 33], where the authors observed no difference in analgesic consumption in the early postoperative period with regard to mesh type. In our study we observed no correlation for interaction between kind of mesh and type of hernia and pain intensity in the perioperative period, which could explain the difference in the analgesic consumption.

The percentage of our patients with chronic pain in the operated groin (13.5\% after 3 months, $9 \%$ after 6 months) is comparable to the incidence rate of $10-30 \%$ of patients reported by other authors [14, 34]. The chronic pain in the groin was more intense for obese and overweight patients as well as those operated on by surgeons other than 1 or 2 . This confirmed the reports of other studies which assessed the effect of body mass, surgeon and technique on results of hernia repair $[8,12,16,17,30,34,35]$. Lower intensity of chronic pain (statistically significant after 6 months) and lower risk of postoperative ailments such as feeling of a foreign body (statistically significant after 3 months) observed in our patients with LW mesh vs. patients with HW mesh is consistent with the results of similar studies [18, 20, 21, 32] but for some authors this effect is true only for a short postoperative period [21-23].
A vast majority of our study patients with both mesh types expressed a high degree of satisfaction with the surgical results and plastic effects of hernia repair. Follow-up after the $6^{\text {th }}$ month showed that full satisfaction with treatment was higher for patients with LW mesh or patients operated on by surgeon 1 or 2 . This confirms the positive effect of the surgeon's experience $[30,31]$ reported by other authors. However, there are also opinions that long-term results of surgery performed by both experienced surgeons and beginners are comparable $[1,35]$. The level of expertise and technical skills of the surgeon often determine the extent of surgical damage and affect further recovery $[9,12,15]$. The results of our study confirm the opinion expressed both by patients and doctors that the Lichtenstein technique is an excellent method of primary inguinal hernia repair $[1,2,7,8,11,15-18,21]$.

\section{Conclusions}

Both the light, partially absorbable mesh and the heavy, non-absorbable mesh used in the Lichtenstein technique are equally effective for prevention of inguinal hernia recurrence. Light, partially absorbable mesh vs. heavy, non-absorbable mesh results in lower intensity of chronic pain, lower risk and intensity of postoperative ailments, faster return to normal activities of daily living and sport as well as higher satisfaction with treatment. The type of mesh has no effect on surgery duration, risk of intraoperative and early postoperative complications, or pain intensity in the early postoperative period. Individual patients' characteristics such as body mass, general condition, type of hernia, surgery duration and operator have a significant effect on the risk of intraoperative damage, percentage of early complications, return to normal activities of daily living, chronic pain, ailments in the operated groin and patient's satisfaction with treatment.

\section{References}

1. Amid PK. Lichtenstein tension-free hernia repair: its background and evolution with focus on avoiding postherniorrhaphy chronic pain. Videosurgery Miniinv 2009; 4: 26-31.

2. Matyja A, Kibil W, Pach R, et al. Assessment of inguinal hernia treatment results in patients operated on with mesh using Lichtenstein, PHS and Robbins-Rutkow techniques. Videosurgery Miniinv 2010; 5: 27-34.

3. Douek M, Smith G, Oshowo A, et al. Prospective randomised controlled trial of laparoscopic versus open inguinal hernia mesh repair: five year follow up. BMJ 2003; 326: 1012-3. 
4. Eklund AS, Montgomery AK, Rasmussen IC, et al. Low recurrence rate after laparoscopic (TEP) and open (Lichtenstein) inguinal hernia repair: a randomized, multicenter trial with 5-year follow-up. Ann Surg 2009; 249: 33-8.

5. Pesta W, Kurpiewski W, Łuba M, et al. Single incision laparoscopic surgery transabdominal pre-peritoneal hernia repair case report. Videosurgery Miniinv 2012; 7: 137-9.

6. McCormack K, Scott NW, Go PM, et al. Laparoscopic techniques versus open techniques for inguinal hernia repair. Cochrane Database Syst Rev 2003; 1: CD001785.

7. Simons MP, Aufenacker T, Bay-Nielsen M, et al. European Hernia Society guidelines on the treatment of inguinal hernia in adult patients. Hernia 2009; 13: 343-403.

8. Bierca J, Kosim A, Kołodziejczak M, et al. Effectiveness of Lichtenstein repairs in planned treatment of giant inguinal hernia own experience. Videosurgery Miniinv 2012; doi: 10.5114/wiitm. 2011.30971.

9. Cueto RR, De Baerdemacker Y, Polliand C, Champault G. Surgical training and inguinal hernia repair. Ann Chir 2006; 131: 311-5.

10. Modrzejewski A, Śmietański M. Postoperative interstitial hernia as a cause of obscure incisional wound site pain. Videosurgery Miniinv 2012; 7: 59-62.

11. Dąbrowiecki S, Pierściński S, Szczęsny W. The Glubran 2 glue for mesh fixation in Lichtenstein's hernia repair: a double-blind randomized study. Videosurgery Miniinv 2012; 7: 96-104.

12. Franneby U, Sandblum G, Nordin O, et al. Risk factors for longterm pain after hernia surgery. Ann Surg 2006; 224: 212-9.

13. Köninger J, Redecke J, Butters M. Chronic pain after hernia repair: a randomized trial comparing Shouldice, Lichtenstein and TAPP. Arch Surg 2004; 389: 361-5.

14. Nienhuijs S, Staal E, Strobbe L, et al. Chronic pain after mesh repair of inguinal hernia: a systematic review. Am J Surg 2007; 194: 394-400.

15. Paajanen H, Varjo R. Ten year audit of Lichtenstein hernioplasty under local anaesthesia performed by surgical residents. BMC Surg 2010; 10: 24.

16. Pielaciński K, Szczepanik AB, Misiak A, Wróblewski T. Randomized clinical trial comparing inguinal hernia repair with Lichtenstein technique using non-absorbable or partially absorbable mesh. Preliminary report. Videosurgery Miniinv 2011; 6: 190-206.

17. Matthews RD, Anthony T, Kim LT, et al. Factors associated with postoperative complications and hernia recurrence for patients undergoing inguinal hernia repair: a report from the VA Cooperative Hernia Study Group. Am J Surg 2007; 194: 611-7.

18. Bringman S, Wollert S, Osterberg J, et al. Three-year results of a randomized clinical trial of lightweight or standard polypropylene mesh in Lichtenstein repair of primary inguinal hernia. $\mathrm{Br}$ J Surg 2006; 93: 1056-9.

19. Erhan Y, Erhan E, Aydede H, et al. Chronic pain after Lichtenstein and preperitoneal (posterior) hernia repair. Can J Surg 2008; 51: 383-7.

20. O'Dwyer PJ, Kingsnorth AN, Molloy RG, et al. Randomized clinical trial assessing impact of a lightweight or heavyweight mesh on chronic pain after inguinal hernia repair. Br J Surg 2005; 92 166-70.

21. Post S, Weiss B, Willer M, et al. Randomized clinical trial of lightweight composite mesh for Lichtenstein inguinal hernia repair. Br J Surg 2004; 91: 44-8.
22. Koch A, Bringman S, Myrelid P, et al. Randomized clinical trial of groin hernia repair with titanium-coated lightweight mesh compared with standard polypropylene mesh. Br J Surg 2008; 95: 1226-31.

23. Bury K, Smietański M, The Polish Hernia Study Group. Five-year results of a randomized clinical trial comparing a polypropylene mesh with a poliglecaprone and polypropylene composite mesh for inguinal hernioplasty. Hernia 2012; 16: 549-53.

24. Klosterhalfen B, Junge $\mathrm{K}$, Klinge U. The lightweight and large porous mesh concept for hernia repair. Expert Rev Med Devices 2005; 2: 103-17.

25. Yin $Y$, Song T, Liao B, et al. Antibiotic prophylaxis in patients undergoing open mesh repair of inguinal hernia: a meta-analysis. Am Surg 2012; 78: 359-65.

26. Sanchez-Manuel FJ, Lozano-García J, Seco-Gil JL. Antibiotic prophylaxis for hernia repair. Cochrane Database Syst Rev 2012; 15: CD003769.

27. Schmedt CG, Sauerland S, Bittner R. Comparison of endoscopic procedures vs. Lichtenstein and other open mesh techniques for inguinal hernia repair: a meta-analysis of randomized controlled trials. Surg Endosc 2005; 19: 188-99.

28. Lindström D, Sadr-Azodi O, Bellocco R, et al. The effect of tobacco consumption and body mass index on complications and hospital stay after inguinal hernia surgery. Hernia 2007; 11: 117-23.

29. Pavlidis TE, Symeonidis NG, Rafailidis SF, et al. Tension-free by mesh-plug technique for inguinal hernia repair in elderly patients. Scand I Surg 2010; 99: 137-41.

30. Lerut T. The surgeon as a prognostic factor. Ann Surg 2000; 232: 729-32.

31. Sandblum G, Sevionius D, Stael von Holstein C. Impact of operative time and surgeon satisfaction on the long-term outcome of hernia repair. Hernia 2009; 13: 581-3.

32. Uzzaman MM, Ratnasingham K, Ashraf N. Meta-analysis of randomized controlled trials comparing lightweight and heavyweight mesh for Lichtenstein inguinal hernia repair. Hernia 2012; 16: 505-18.

33. Nikkolo C, Lepner U, Murruste M, et al. Randomised clinical trial comparing lightweight mesh with heavyweight mesh for inguinal hernioplasty. Hernia 2010; 14: 253-8.

34. Poobalan AS, Bruce J, King PM, et al. Chronic pain and quality of life following open inguinal hernia repair. Br I Surg 2001; 88: 1122-6.

35. Massaron S, Bona S, Fumagalli U, et al. Analysis of post-surgical pain after inguinal hernia repair: a prospective study of 1,440 operations. Hernia 2007; 11: 517-25.

Received: 5.11.2012, revised: 12.11 .2012 , accepted: 3.12.2012. 